\title{
Measurement on the SRRC 1.3 GeV Electron Booster Synchrotron Operation Parameters and the Ramping Behavior
}

\author{
K. K. Lin, K. T. Hsu, T. S. Ueng \\ Synchrotron Radiation Research Center \\ No. 1, R \& D Road IV, Hsinchu Science-Based Industrial Park \\ Hsinchu 30077, Taiwan, R. O. C.
}

\begin{abstract}
The booster operation parameters, such as synchrotron tune, betatron tune, momentum compaction factor, betatron function etc.were measured at injection energy, $50 \mathrm{MeV}$, and compared with the calculated results. The synchrotron tune shift was done by varying the applied cavity voltage, and the betatron tune shift was observed by changing the current on the quadrupole magnets. The measured results of booster beam emiltance and energy spread during acceleration were based on the beam size measurement from the emitted synchrotron light. Possible maximum emittance and energy spread were obtained directly from the measured data, assuming the corresponding calculated betatron and dispersion functions at the point of observation were correct. The measured results were also compared with the theoretical expection.
\end{abstract}

\section{INTRODUCTION}

The SRRC booster was manufactured and delivered by Scanditronix AB, Sweden in July 1992 [1]. The booster rf system was provided by the SRRC if group. In order to understand the booster performance characteristics, the operation parameters were measured at various aspects and compared with the designed values.

\section{OPERATION PARAMETERS}

These experiments were carried out at injection energy, 50 $\mathrm{MeV}$, with energy spread set at $+1-0.5 \%$.

\section{Synchrotron Tune and Momentum Compaction Factor}

The electron beam signal was picked-up with a stripline monitor and analyzed with a spectrum analyzer. Synchrotron tune was measured by observing the sideband of the revolution signal. Using the following relation, the momentum compaction factor was determined to be 0.57 compared with the calculated 0.58 .

$$
\begin{aligned}
& \mathrm{f}_{\mathrm{S}}^{2}=\mathrm{f}_{\mathrm{r}}^{2} \text { [e.h.cosf } f_{\mathrm{S}} \cdot \alpha \cdot \mathrm{V}_{\mathrm{rf}} /[2 \pi \mathrm{E}] \text {, where } \\
& \mathrm{f}_{\mathrm{S}}: \text { synchrotron oscillation frequency; } \\
& \mathrm{f}_{\mathrm{r}}: \text { revolution frequency, } 4.167 \mathrm{MHz} ; \\
& \mathrm{e}: \text { electron charge; } \\
& \mathrm{h}: \text { harmonic number, } 120 ; \\
& \mathrm{f}_{\mathrm{S}}: \text { synchronous phase; } \\
& \mathrm{V}_{\mathrm{rf}}: \text { peak rf voltage; } \\
& \text { E: electron beam energy; } \\
& \alpha: \text { momentum compaction factor. }
\end{aligned}
$$

The measured result is shown in figure 1. The experimental uncertainty were few percent on $\mathrm{E}$ and about $10 \%$ on both $\mathrm{f}_{\mathrm{S}}$ and $V_{\text {rf. }}$

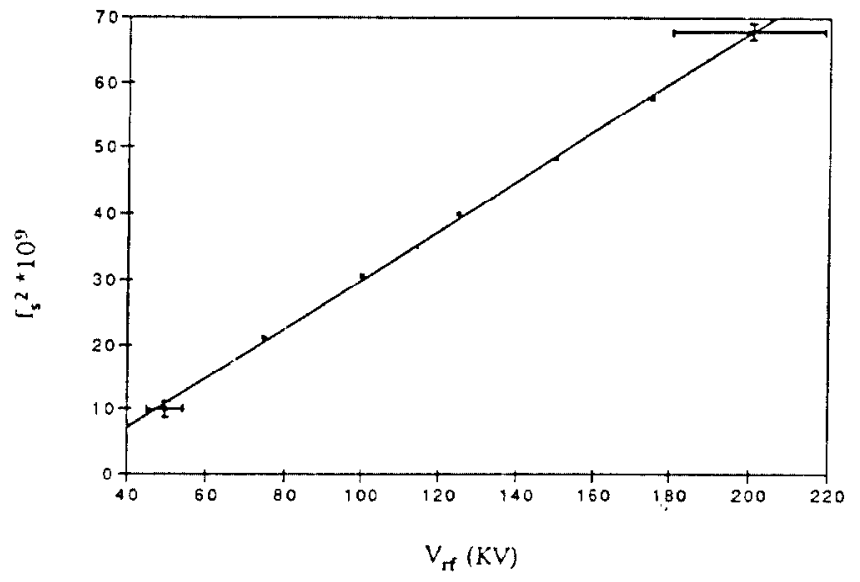

Figure 1. Synchrotron frequency verses rf cavity gap voltage. The fitted slope is $3.77 \exp (5)$

\section{Betatron Tune and Tune Diagram}

A tracking generator was used as an excitation source in order to perturb the beam for betatron tune measurement. The betatron tune was first set to the designed value, then both focusing and defocusing quadrupole families were adjusted to look for the tuning range. Typical display on the spectrum analyzer is shown in figure 2 , with $V_{X}=4.4, V_{z}=2.43$. The uncertainty in the tune fractional part was estimated to be 0.02 .

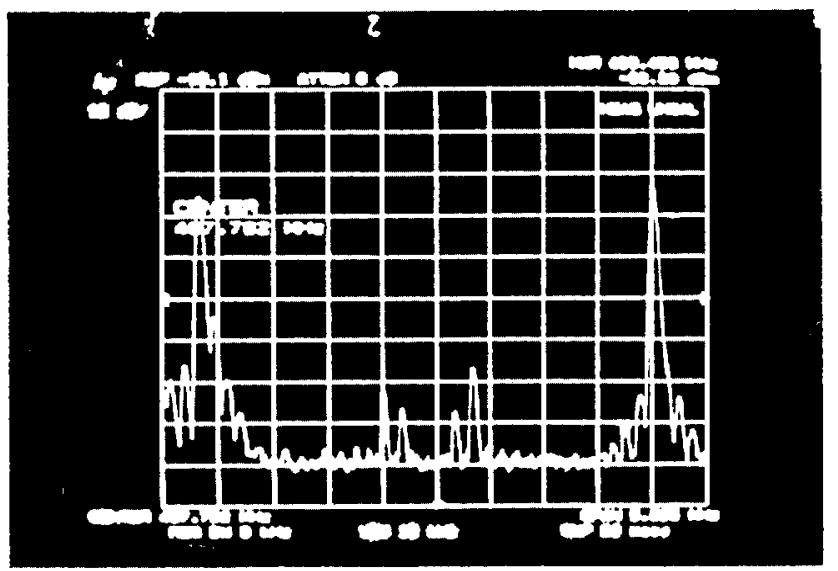

Figure 2. Typical operation tune signal. The highest peaks on both sides are the revolution signal of $4.167 \mathrm{MHz}$ apart. Two pairs of secondary peaks around the ccnter are the betatron tune signals of $v_{X}=4.4, v_{Z}$ $=2.43$. The horizontal scale is $0.5 \mathrm{MHz} / \mathrm{div}$. 
By using the associated quadrupole strengths in the experiment, the measured ones agree with the calculated tunes from MAD [2] or PATRICIA [3]. The observed tuning range for $V_{x, z}$ were

$$
\begin{aligned}
& 4.33<v_{X}<4.44, \text { and } \\
& 2.33<v_{z}<2.44 .
\end{aligned}
$$

The corresponding tune diagram is given in figure 3 , and the indicated resonant lines satisfy

$$
m v_{x}+n v_{z}=k
$$

where $\mathrm{m}, \mathrm{n}$, and $\mathrm{k}$ are integers. The measured tuning range shows that the beam survives while crossing the coupling resonant line and was limited by the stop band on $v_{x, z}$.

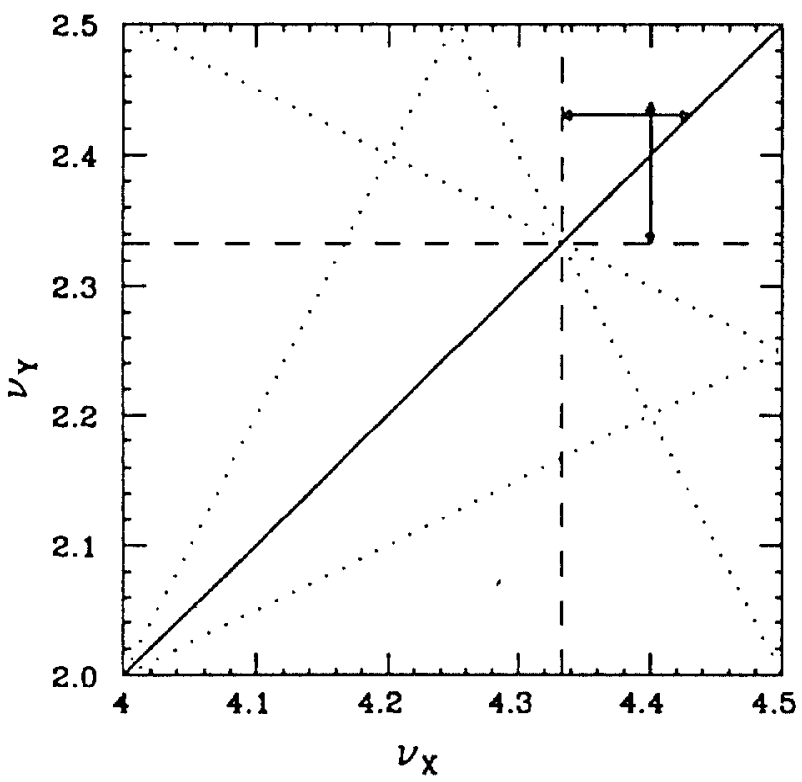

Figure 3. The tune diagram of the booster. Tunning range for both $v_{\mathrm{X}}$ and $v_{\mathrm{Z}}$ are also indicated.

\section{Betatron Functions}

The betatron functions were measured by changing the quadrupole current and observing the associated tune shift. The measurement was carried out with operating tune $V_{X}=$ $4.4, v_{z}=2.43$. The lattice in one superperiod of the booster consists of one bending magnet, two quadrupoles (FQ, DQ), and two correction magnets. The correction magnet consists of steering, quadrupole, and scxtupolc windings. The measurement of betatron function at certain location was done by adjusting the strength of each quadrupole families and observing the associated tune shifts. This gave an averaged betatron function at the quadrupole locations. Also, by changing the individual quadrupole component driving current in the correction magnet and observing the corresponding tune shifts, betatron function at that particular corrector location can be obtained. The measured betatron function was determined from the following relation,

$$
\Delta V_{x, z}=(1 / 4 \pi) \cdot \beta_{x, z} \cdot k \cdot 1 \cdot(\Delta l / I),
$$

where $\Delta V$ is the observed tune shift, $k$ stands for the quadrupole strength, 1 represents the magnet effective length, and $\Delta I$ is the current change which moves the tune. The measured results in two consequtive sections of the booster is also shown in figure 4 . The major cause of the measurement uncertainty is due to the tune shift estimation. In figure 4 , for those data which give high beta values, the uncertainty is in the range of $25 \%$. For low beta values, the uncertainty is about $80 \%$.

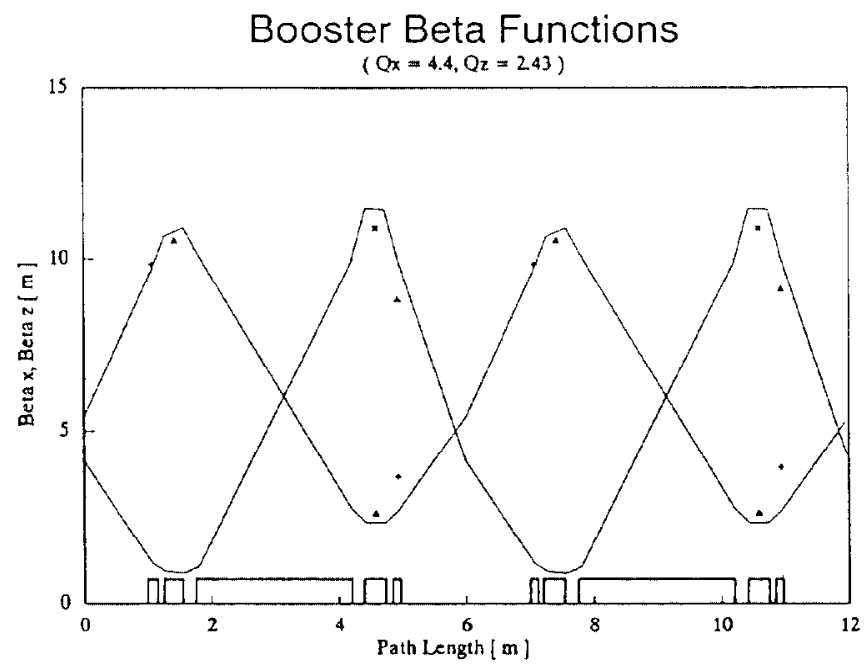

Figure 4. Both calculated and measured betatron functions in two consecutive superperiods.

\section{RAMPING BEHAVIOR}

\section{Beam size, Horizontal Emittance, and Energy Spread}

The measured beam emittance and energy spread during acceleration were based on the beam size measurement from the emitted synchrotron light, assuming the corresponding calculated betatron and dispersion functions at the point of observation were correct. The measured results were compared with the theoretical expection.

The enitted synchrotron light was collected by a CCD camera with resolution of $22 \mu \mathrm{m}$ in vertical and $11 \mu \mathrm{m}$ in horizontal directions. It is assumed that the measured photon spot size is the same as the corresponding electron beam size in this case [4]. Both beam emittance $(E)$ and energy spread $(\Delta E / E)$ contribute to the beam size $(\sigma)$ with the relation

$$
\sigma=\left[\varepsilon \cdot \beta+(\eta \cdot \Delta \mathrm{E} / \mathrm{E})^{2}\right]^{1 / 2},
$$

where $\beta$ and $\eta$ are the corresponding betatron and dispersion functions at the emission source respectively.

During the acceleration period, the competition among processes such as adiabatic damping, radiation damping, and quantum excitation leads to ever changing values for dynamic parameters of the electron beam as a function of time. The measured beam emittance and beam energy spread variation during acceleration period are shown in figure 5 and figure 6 together with the theoretical expectation [5]. In figure 5, at every measurement step, the emittance was obtained by making use of the calculated lattice parameters, such as $\Delta E / E$ at that particular energy, and the calculated $\beta$ and $\eta$. The upper limit of the emittance $\left(\varepsilon_{\max }\right)$ was obtained by assuming that emittance was the only contribution factor to 
beam size. The experimental results show that the adiabatic and radiation dampings give major contribution to emittance damping in the early stage of acceleration, while quantum excitation effect is responsible for emittance growth as the beam energy becomes higher. The contract required emittance specifiction is also indicated in the figure. In figure 6 , it also indicates that the energy spread variation is caused by similar damping processes as have been described above.

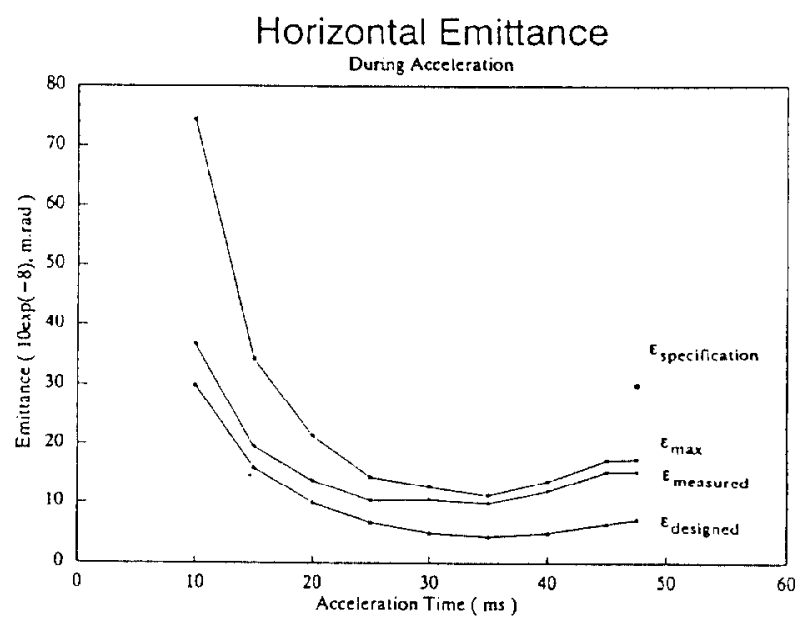

Figure 5. Emittance variation as a function of acceleration time. Ramping period is $50 \mathrm{~ms}$. Repetition rate is $10 \mathrm{~Hz}$.

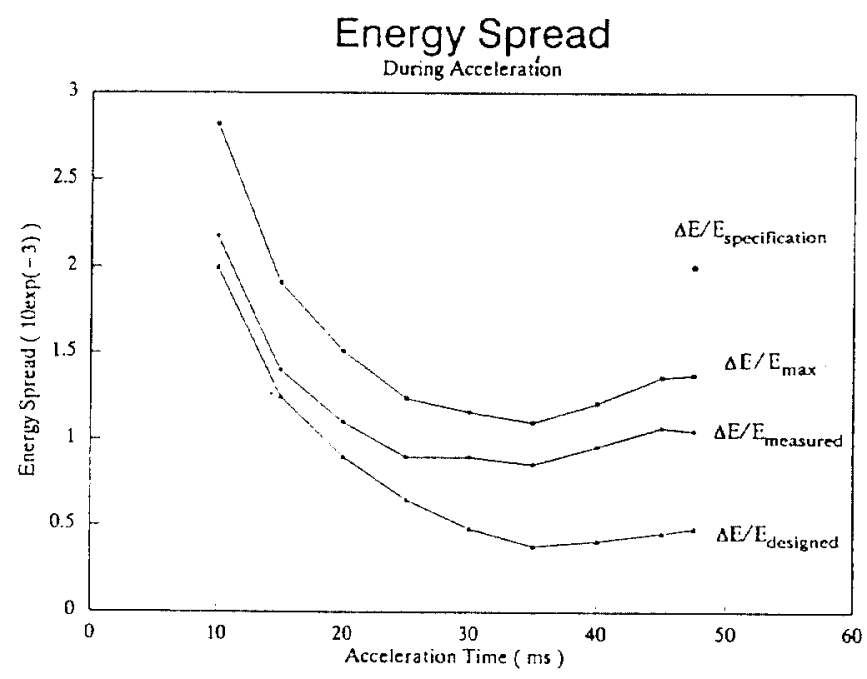

Figure 6. Energy spread variation during ramping period.

\section{REFERENCES}

[1] J. Modeer, project manager, Scanditronix AB, "1.3 GeV Electron Synchrotron" in this conference, $\mathrm{Pb} 12$.

[2] F. C. Iselin, The MAD Program Reference Manual, CERN-LEP-TH/85-15, May 1985.

[3] H. Wiedemann, Users Guide for Patricia version 85.5, SSRL ACD-NOTE 29, May 1985.

[4] A. Hofmanu, F. Meot, Nuclear Instruments and Methods 203(1982)483-493.

[5] (a) K. K. Lin, K. C. Cheng. Nucl. Sci. J. 25(4), p.227-246, August, 1988. (b) Scanditronix AB, Conceptual Design Report of a $1.3 \mathrm{GeV}$ Electron Beam Injection System for the Synchrotron Radiation Research Center, December, 1988. (c) K. K. Lin, K. C. Cheng. SRRC/IJ/IM/89-01. Rev.(1), January, 1989. 